\title{
Práctica Experimental de Disección y Modelación 3D de Oído Medio e Interno para la Construcción Significativa de Conocimiento en el Área de Anatomía Humana
}

\author{
Experimental Practice of Dissection and 3D Modeling of the Middle and \\ Inner Ear for the Development of Increased Knowledge of Human Anatomy
}

Sonia Osorio Toro

OSORIO, T. S. Práctica experimental de disección y modelación 3D de oído medio e interno para la construcción significativa de conocimiento en el Área de Anatomía Humana. Int. J. Morphol., 38(4):997-1002, 2020.

RESUMEN: La comprensión de la anatomía humana y especialmente de algunos órganos muy pequeños como es el oído medio e interno, resulta desafiante debido a su compleja estructura tridimensional. Construir conocimiento en la comprensión de la Anatomía del oído medio e interno humano, a partir del experimento de disección y modelación 3D. Con imágenes DICOM de una tomografía axial computarizada del oído humano convertidas a un modelo 3D (Mimics) y disecciones cadavéricas del hueso temporal, se diseñaron las estructuras óseas y blandas del oído externo, medio e interno mediante la implementación de software de modelado orgánico. Creación de un protocolo operativo estándar, modelado 3D de oído medio e interno e impresión 3D de las estructuras, diferenciando por color cada una de ellas. La experimentación, en este caso, la disección y modelación de las piezas anatómicas fue fundamental en la enseñanza y aprendizaje de esta área de las ciencias, permitiendo que se construya conocimiento a partir de la búsqueda de información, observación, análisis y reflexión. Los estudiantes manifestaron fascinación y aprendizaje profundo en la práctica realizada. La construcción de modelos explicativos en torno a la experimentación cualitativa permite comprender a profundidad los diferentes fenómenos. La actividad experimental, supera la demostración y facilita la confrontación de pensamiento, los estudiantes a través de esta actividad comprendieron a profundidad la anatomía del oído medio e interno, han adquirido destrezas quirúrgicas, han propuesto y analizado proyectos que les ha permitido participar eventos académicos, adquiriendo competencias como el uso comprensivo del conocimiento científico.

PALABRAS CLAVE: Oído; Anatomía; Disección, Enseñanza-Aprendisaje; Anatomía Humana.

\section{INTRODUCCIÓN}

La enseñanza de la Anatomía Humana ha cambiado a lo largo de la historia, pasando por la explicación de las piezas a través de la comparación con la anatomía animal, la disección, la observación de órganos in vivo (endoscopias, radiografías, tomografías, etc), la plastinación, hasta el actual uso de las tecnologías, como son los software 3D, realidad aumentada, etc. (Araujo Cuauro, 2018), anteriormente en los cursos se incluía el dibujo anatómico como parte importante dentro de la asignatura, esta actividad se ha eliminado de los programas y la disección que permite el reconocimiento de las estructuras y la comprensión de la tridimensionalidad de los órganos cada vez es más difícil, debido a la restricción a los cadáveres, sin embargo, se considera que esta actividad proporciona un conocimiento anatómico completo y puede mejorar las habilidades quirúrgicas (Anschuetz et al., 2018).
Actualmente, a pesar de los diversos avances tecnológicos la forma de enseñar en algunas universidades la Anatomía Humana es de forma expositiva, magistral y la práctica en muchas ocasiones se convierte en clase magistral nuevamente, el docente explica las diferentes regiones en la clase teórica con ayuda de dibujos bidimensionales y posteriormente en la práctica suele dar el discurso nuevamente con la pieza real o con el modelo anatómico; Generalmente se utilizan guías de práctica en las que se especifica el paso a paso de lo que se debe hacer, de lo que se debe observar, así como Atlas de Anatomía con imágenes bidimensionales en diferentes planos y vistas que utilizan colores de forma didáctica que al ser contrastados con las piezas reales difieren en la forma, el tamaño, la ubicación, la textura y no es posible comprender cuando se es principiante la tridimensionalidad. 
OSORIO, T. S. Práctica experimental de disección y modelación 3D de oído medio e interno para la construcción significativa de conocimiento en el Área de Anatomía Humana. Int. J. Morphol., 38(4):997-1002, 2020.

Dentro de las estructuras anatómicas humanas de mayor complejidad se encuentra el oído medio e interno, puesto que sus piezas son muy pequeñas por lo que no es posible visualizarlas en el cadáver y los modelos anatómicos que se encuentran no corresponden fielmente a la realidad; Esta región ha sido descrita como difícil de enseñar por sus relaciones tridimensionales, una forma de mejorar la comprensión de sus estructuras es utilizando la tecnología 3D ( $\mathrm{Ng}$ et al., 2015).

La enseñanza de la Anatomía del oído humano ha sido problemática puesto que la explicación y los materiales de estudio más utilizados no logran promover comprensión y significado en las estudiantes. No obstante, el progreso de la Medicina en general se ha debido en gran parte al trabajo experimental, la investigación biomédica en general ha trabajado en diferentes experimentos, especialmente con biomodelos con el fin de aplicar nuevas técnicas quirúrgicas, demostración y adquisición de habilidades, pruebas diagnósticas, elaboración de vacunas y enseñanza; Las áreas de la salud exigen un conocimiento riguroso del origen y evolución de las enfermedades, los medios terapéuticos y su correcta aplicación, el experimento favorece la formación del pensamiento crítico en los estudiantes en formación (Garza-Rodea et al., 2007).

Los nuevos filósofos e historiadores rescatan el papel del experimento cualitativo en la construcción del conocimiento (García \& Estany, 2010). La disección y modelación 3D vistos como experimento, permiten la interacción de los estudiantes con las piezas anatómicas, tejiendo conocimiento y dotándolos de significado. Esta comprensión no es posible leyendo libros de texto, observando imágenes bidimensionales y asistiendo a clases magistrales sobre el tema. La disección del oído medio e interno constituye un método completo en el estudio de la anatomía dado que permite apreciar sus dimensiones y configuración real, así como posibles variaciones anatómicas. La disección es considerada por los estudiantes como una herramienta educativa valiosa y útil para enseñar y aprender esta área del conocimiento (Flack \& Nicholson, 2017).

La estructura retórica de los libros de texto de Anatomía Humana, como la mayoría de libros que se utilizan en la educación superior, no contribuyen con la comprensión de los estudiantes, más bien pueden aumentar la dificultad (García \& Estany). Generalmente las descripciones utilizadas son muy detalladas, no hay un orden en los temas para hacerlo de manera lógica, pedagógica y progresiva, en todos los capítulos se mezclan diferentes temas y se utiliza una terminología especializada, de tal manera que para alguien que se encuentra aprendiendo, necesitaría apoyo en la lectura, debido a que la capacidad de leer y escribir de los estudiantes está relaciona- da con las formas de acceso a la cultura y con los métodos de enseñanza de la lengua materna (Leme Britto, 2003).

El conocimiento presentado en los libros de Anatomía ha sido de modelos teóricos, que son explicaciones al mundo real basados en hipótesis y conjeturas que recogen los hechos como elemento fundamental, las disecciones se realizan siguiendo guías específicas, considerando este experimento como verificador de la teoría, tal como lo consideró el empirismo lógico; Es importante considerarlo como constructor de conocimiento científico, que involucre problemáticas y que tenga una riqueza conceptual en sí mismo (García, 2011). Teniendo en cuenta que la relación entre la teoría y el experimento ha sido diversa, se propone construir conocimiento en la comprensión de la Anatomía del oído medio e interno humano, a partir del experimento de disección y modelación 3D.

\section{MATERIAL Y MÉTODO}

Este trabajo se enmarca dentro del proyecto titulado Recurso Educativo Abierto para el aprendizaje de la Anatomía humana, que cuenta con el aval del Comité Institucional de Revisión de Ética Humana con código interno 121-018 del año 2018.

Se realizó un experimento de tipo cualitativo en 3 cadáveres humanos que se encuentran en estado de conservación en el Anfiteatro de la Universidad del Valle, utilizando referentes anatómicos para delimitar y aislar una estructura paralelepípeda, se extrajeron los peñascos del hueso temporal obteniendo seis especímenes sobre los cuales se realizó disección con osteotomía utilizando fresa 703 y redondas HP 2,4 y 8, con Mototool. Mediante osteoplastia se logró delimitar el contorno del laberinto óseo del oído interno articulado con la cadena de huesecillos.

A partir de ensayo y error, se elaboró un procedimiento operativo estándar que permitiera tener referentes anatómicos estables, que explicaba el paso a paso de la disección, incluyendo la demarcación de los cuadrángulos para extraer la pieza ósea, el reconocimiento de las estructuras anatómicas por cada una de las caras (anterior, posterior, medial, lateral, superior e inferior), la explicación de las técnicas de abordaje y la elaboración de esquemas didácticos (Fig. 1).

Con imágenes DICOM de una tomografía axial computarizada del oído humano convertidas a un modelo 3D (Mimics) y las disecciones cadavéricas del hueso temporal, se diseñaron las estructuras óseas y blandas del oído externo, medio e interno mediante la implementación de software de modelado orgánico. 


\section{RESULTADOS}

La elaboración del procedimiento operativo estándar permitió describir de manera detallada y sistemática cada uno de los pasos que se realizaron, se tomaron fotografías de las pequeñas piezas que componen este órgano, explicando minuciosamente su ubicación, se logró aislar el oído medio e interno para su estudio. Se examinó el trayecto de los nervios facial, vestibulococlear, petroso mayor, entre otros, se delimitó la cadena de huesecillos articulada con sus músculos y demás estructuras blandas asociadas. Por último, se estudiaron los detalles y forma del laberinto óseo, así como también su configuración interna.

Se realizaron esquemas de representación de las estructuras observadas con ayuda de un Diseñador Gráfico y con imágenes DICOM de una tomografía axial computarizada del oído humano convertidas a un modelo 3D (Mimics) y las disecciones cadavéricas del hueso temporal, se diseñaron las estructuras óseas y blandas del oído externo, medio e interno mediante la implementación de software de modelado orgánico (Autodesk Maya, Zbrush , 3 Matic). El modelo final fue materializado en ácido poliláctico (PLA) por una impresora en 3D (Prusa), con ampliación de la escala 1:10 y la utiliza-

\section{Oído Izquierdo}
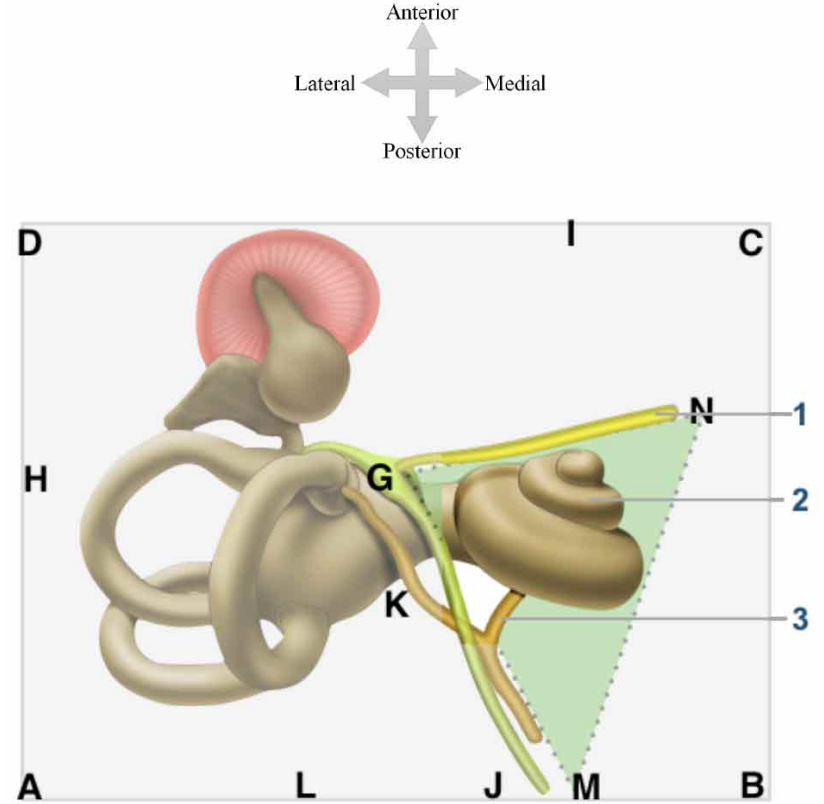

A1 ción de códigos de color para las estructuras. La decisión de utilizar modelado 3D se debe a que en la literatura se encuentra que éstas herramientas mejoran la comprensión de las estructuras ( $\mathrm{Ng}$ et al.).

Como resultado del trabajo interdisciplinar (Estudiantes de Medicina e Ingeniería Biomédica), se diseñó e imprimió en 3D un modelo del oído que respeta las proporciones y el emplazamiento de las estructuras óseas y blandas dentro del hueso temporal. Tanto el diseño virtual en 3D, compartido en la plataforma abierta Sketchfab, como el impreso en códigos de color, incluyen: los nervios facial, vestibulococlear y petroso mayor; el laberinto óseo y membranoso; la cadena de huesecillos; la membrana timpánica; los músculos tensor del tímpano y estapedio, así como los conductos auriculares interno y externo, el pabellón auricular y la arteria carótida interna en su porción petrosa (Fig. 2).

https://sketchfab.com/3d-models/estructuras-del-oido 0492b4cd0c134afb93d8a091d141930c (Link para acceder al modelo virtual)

\section{Oído Derecho}
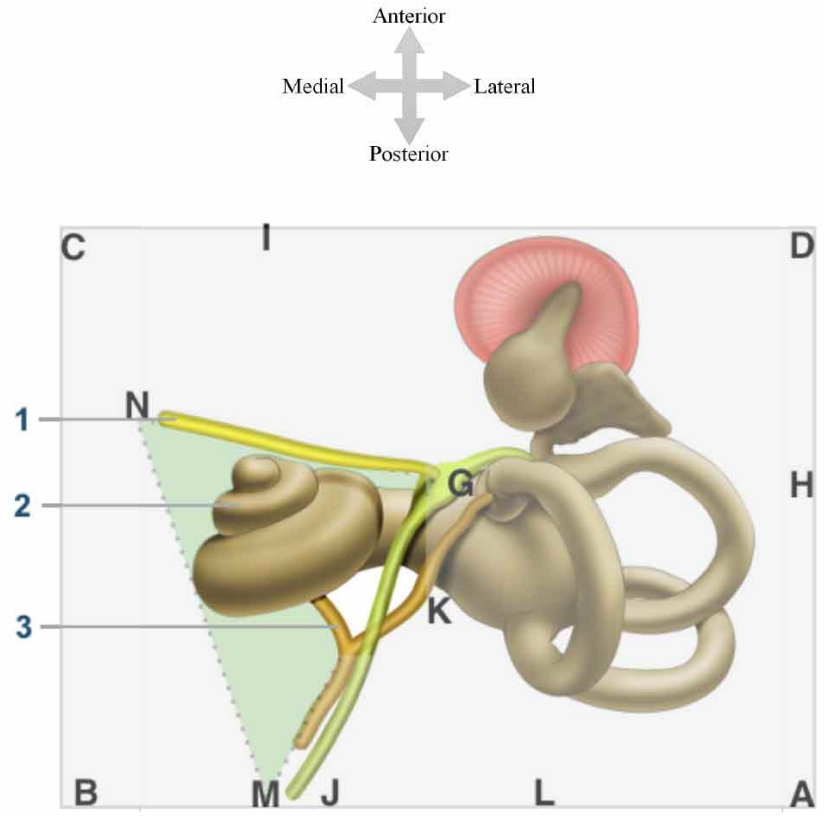

A

Fig. 1: Gráfico Porción Petrosa del hueso Temporal PPT- Cara superior (cuadrángulo A-B-C-D), vista superior. Área: G-M-N cóclea, 1: Nervio Petroso Mayor, 2: Cóclea, 3: Nervio Coclear. A1: izquierda. A2: derecha. 

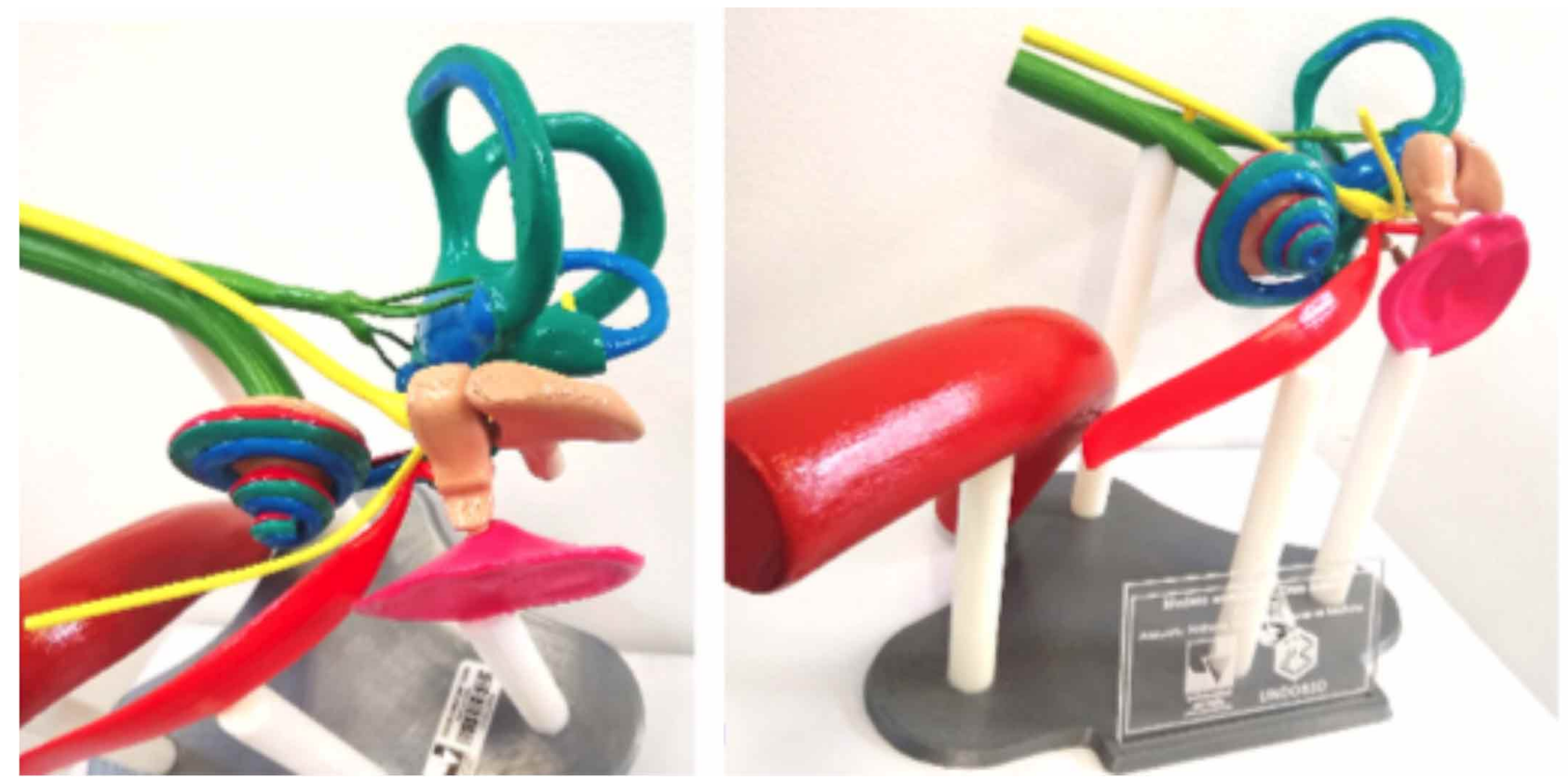

Fig. 2. Impresión 3D de oído medio e interno

\section{DISCUSIÓN}

En el siglo XXI como en todas las áreas del conocimiento se encuentra que los avances tecnológicos relacionados con redes, herramientas y dispositivos para la gestión de la información ha permitido que los docentes consideren y exploren los posibles usos de las tecnologías de la información y la comunicación (TIC) como apoyo en los procesos de enseñanza-aprendizaje, se encuentran diversas aplicaciones que permiten visualizar las estructuras anatómicas de forma tridimensional, es posible disecar virtualmente sin temer a dañar las piezas. Algunas universidades han apostado a la enseñanza de la Anatomía utilizando sólo recursos virtuales y modelos anatómicos de diferentes materiales, aduciendo que no es necesario interactuar con cadáveres para aprender sobre ella, por el contrario otros autores piensan que la realidad virtual palidece ante la experiencia real de abrir un cadáver, puesto que no se aprende sólo anatómica fría como el número de huesos o arterias, sino que lo que queda aprendido y siempre recordado es la realidad compleja de un cuerpo que hace poco estaba vivo (Rovetto, 2008).

La pregunta que se hacen los diferentes profesores del área es la siguiente ¿Realmente necesitamos cadáveres para aprender anatomía en el pregrado?, la respuesta después de realizar un simposio en el que participaron expertos del tema fue que existe una variedad de técnicas de enseñanza, que pueden aportar al conocimiento anatómico, como son la pintura corporal, la examinación clínica y la tecnolo- gía 3D, sin embargo, la disección es preferida por los estudiantes porque enseña una anatomía autentica, que implica una identificación diferencial de estructuras a través de la identificación de texturas de los tejidos y el análisis de las relaciones espaciales (McMenamin et al., 2018).

Así mismo, en una revisión crítica de la literatura se expone que la mejor manera de enseñar anatomía moderna es combinando recursos pedagógicos para complementarse entre sí, los estudiantes aprenden más cuando se integran de manera efectiva enfoques multimodales, dependiendo del pregrado que se curse, la disección es ideal para estudiantes del programa académico de Medicina, especialmente aquellos con intenciones de realizar especialidades quirúrgicas (Estai \& Bunt, 2016).

En este estudio, los estudiantes que participaron valoran la posibilidad de experimentar recibiendo apoyo de la institución, de los profesores y el no sentir presión por una nota, cuando hablan de la experiencia expresan que "Fue increíble descubrir como un niño cada parte, guiados por un libro, fue fascinante. Gracias a este experimento, comprendimos a profundidad la anatomía del oído medio e interno, aprendimos el manejo de herramientas quirúrgicas, trabajamos en la escritura de textos y nos ha permitido presentaciones nacionales e internacionales". Los Médicos en formación han trabajado en el proyecto por 3 semestres, si- 
guiendo los pasos explicados en la metodología. La interacción con el modelo 3D conduce a una mejor comprensión de la forma y relaciones espaciales entre estructuras y permite ilustrar las variaciones anatómicas, complementando la enseñanza, especialmente de regiones anatómicas complejas (Pujol et al., 2016).

De igual manera, hablar de experimentación y/o experiencia es todo un elemento organizador de pensamiento, los estudiantes llegan a través de la experiencia común a una aducción, parten de preguntas, de dudas. Empiezan a caracterizar las piezas que observan y desde ahí empiezan a hacer preguntas, a proponer métodos de exploración, los dibujos bidimensionales realizados se hicieron en colaboración con un diseñador gráfico y la modelación $3 \mathrm{D}$ con ingeniero biomédico, está interacción entre diferentes disciplinas permite resolver problemas complejos y producir desarrollos innovadores, el diseño del aprendizaje multidisciplinario se basa en el problema elegido, en este caso la construcción de herramientas didácticas para el aprendizaje de la anatomía por parte de los estudiantes de Medicina, propicio la integración y la alineación constructiva (Klaassen, 2018).

Hoy en día, se afirma que se ha llegado al fin de la historia de la Anatomía Humana, sin embargo la fragilidad de la salud y la complejidad de la enfermedad humana nos lanzan siempre en humildes búsqueda de nuevos paradigmas. En el pensamiento popperiano la ciencia avanza de falsabilización en falsabilización hasta inciertas verdades nunca finales (Rovetto).

En este sentido, se hace necesario seguir explorando nuevas formas de enseñanza, aprendizaje y evaluación de la Anatomía Humana, buscar enfoques integradores en los que se favorezcan el aprendizaje profundo; la actividad experimental guiada por preguntas problematizadoras invita a pensar con voz propia, no ofrece respuestas definitivas, propicia la búsqueda, el análisis, el pensamiento y la argumentación; No puede haber conocimiento si no se construye la experiencia.

Existen contradicciones sobre cuáles son los mejores métodos que se deben utilizar en el aula; Generalmente se enseñan conceptos, pero la comprensión que se tenga de ellos, es la interpretación que se realice del mismo a partir de la experiencia asociada. Semestralmente se encuentran estudiantes que se fascinan con la actividad de disección, estudian horas extras, prefieren el contacto con las piezas para comprender la estructura, otros, no toleran estar dentro de un anfiteatro y prefieren actividades virtuales para comprender los temas tratados en clase; la mejor opción podría ser realizar disección de piezas cadavéricas humanas, de piezas animales y por medio de la tecnología disección de piezas virtuales; es necesario combinar los métodos tradicionales con la tecnología, puesto que los avances tecnológicos representan una oportunidad para enriquecer y transformar los procesos de enseñanza - aprendizaje y comprender que existe

\section{CONCLUSIONES}

Es posible reorientar el papel que se le da a la experimentación, puesto que contiene una riqueza conceptual muy importante, los estudiantes a través de esta actividad comprendieron a profundidad la anatomía del oído medio e interno, han adquirido destrezas quirúrgicas, han propuesto y analizado proyectos que les ha permitido participar en eventos académicos, adquiriendo competencias como el uso comprensivo del conocimiento científico, capacidad para realizar indagaciones de carácter formal y capacidad para dar explicaciones a fenómenos.

Modelos anatómicos 3D elaborados por estudiantes como alternativas de disección, representan una ventajosa herramienta de aprendizaje en el estudio anatómico del oído, en tanto que permite el acceso de más estudiantes a una representación fidedigna y facilita tanto la comprensión de las proporciones como la ubicación de las diferentes estructuras dentro del hueso temporal.

Analizando los diferentes artículos, libros y de acuerdo a la experiencia docente se puede inferir que históricamente se ha aprendido Anatomía Humana a través de la observación y posterior manipulación de las diferentes estructuras, las imágenes han jugado un papel protagónico en el aprendizaje de ésta, se recomienda el estudio descriptivo y detallado de un libro de texto, siempre acompañado de un atlas que facilite el entendimiento de la posición de los órganos y su relación entre ellos, es posible utilizar y realizar esquemas, dibujos y/o modelos para facilitar la comprensión de la tridimiensionalidad, la profundidad, la forma, el tamaño y las características de cada pieza estudiada, la participación en proyectos de investigación en los cuales se involucren casos clínicos interesantes y de mediana complejidad que los acerque a su quehacer, les permitirá a los estudiantes realizar representaciones mentales que les ayudarán en la comprensión de los diferentes temas tratados.

Los experimentos cualitativos o exploratorios son el inicio de todo tipo de conocimiento que puede llegar hasta la abstracción, la actividad experimental, supera la demostración y facilita la confrontación de pensamiento, el ejercicio de poner en juego la observación permite encontrar los fenómenos y trabaja la capacidad de cuestionarse. 
Adicionalmente, estas actividades experimentales proveen a los estudiantes mayor dominio del instrumental quirúrgico y mejora de las habilidades motoras finas, lo que aportará a la futura práctica quirúrgica; Este método sirve de base para realizar intervenciones in vivo y contrasta en su utilidad para el estudio más global de las estructuras del oído.

AGRADECIMIENTOS. Un agradecimiento especial a la Profesora Dra. Janneth Zúñiga del Departamento de Morfología de la Universidad del Valle, a los estudiantes del Programa Académico de Medicina y Cirugía Natahlie Torres y Wilmer Moreno, por su apoyo en la elaboración del modelo y a la Comisión de Estudios No 096 del 4 de julio de 2019, otorgada por la Universidad del Valle, Cali, Colombia.

OSORIO, T. S. Experimental practice of dissection and 3D modeling of the middle and inner ear for the development of increased knowledge of human anatomy. Int. J. Morphol., 38(4):997-1002, 2020.

SUMMARY: Understanding human anatomy, and especially some very small organs such as the middle and inner ear, is challenging due to their complex three-dimensional structure. To build knowledge in understanding of anatomy, of the human inner and middle ear, from the 3D modeling and dissection experiment. Using DICOM images from a computerized axial tomography of the human ear converted to a 3D model (Mimics) and cadaveric dissections of the temporal bone, the bony and soft structures of the external, middle, and internal ear were designed, using organic modeling software. A standard operating protocol, 3D modeling of the middle and inner ear and 3D printing of the structures was created, differentiating each one by color. In this case, the dissection and modeling of the anatomical pieces was fundamental in the teaching and learning of this area of science, allowing expanded knowledge from the search for information, observation, analysis and reflection. The students showed fascination and deep learning in the practice carried out. The construction of explanatory models around qualitative experimentation allows an in-depth understanding of the different phenomena. The experimental activity overcomes the demonstration and facilitates thought process. The students achieve and in depth understanding of the middle and inner ear anatomy, acquired surgical skills, proposed and analyzed projects that allowed them to participate in academic event. They have also acquired additional skills, such as the comprehensive use of scientific knowledge.

KEY WORDS: Ear; Anatomy; Dissection.

\section{REFERENCIAS BIBLIOGRÁFICAS}

Anschuetz, L.; Presutti, L.; Marchoni, D; Bonali, M.; Wimmer, W.; Villari, D. \& Caversaccio, M. Discovering middle ear anatomy by transcanal endoscopic ear surgery: a dissection manual. J. Vis. Exp., (131), 2018. DOI: https://doi.org/10.3791/56390

1002
Araujo Cuauro, J. C. Aspectos históricos de la enseñanza de la anatomía humana desde la época primitiva hasta el siglo XXI en el desarrollo de las ciencias morfológicas. Rev. Argent. Anat. Online, 9(3):87-97, 2018.

Estai, M. \& Bunt, S. Best teaching practices in anatomy education: A critical review. Ann. Anat., 208:151-7, 2016.

Flack, N. A. M. S. \& Nicholson, H. D. What do medical students learn from dissection? Anat. Sci. Educ., 11(4):325-35, 2017.

García, A. E. G. \& Estany, A. Filosofía de las prácticas experimentales y enseñanza de las ciencias. Prax. Filos., (31):7-24, 2010.

García, A. E. Modelos de explicación, basados en prácticas experimentales. Aportes de la filosofía historicista. Rev. Cient., (14):89-96, 2011.

Garza-Rodea, A. S.; Padilla-Sánchez, L.; Garza-Aguilar, J. \& Neri-Vela, R. Algunas notas sobre la historia del laboratorio de cirugía experimental. Reflexiones sobre su importancia en la educación e investigación quirúrgica. Cir. Cir., 75(6):499-505, 2007.

Klaassen, R. Interdisciplinary education: a case study. Eur. J. Eng. Educ., 43(6):842-59, 2018

Leme Britto, L. P. La cultura escrita y la formación del estudiante universitario. Lenguaje, (31):78-92, 2003.

McMenamin, P. G.; McLachlan, J.; Wilson, A.; McBride, J. M.; Pickering, J.; Evans, D. J. R. \& Winkelmann, A. Do we really need cadavers anymore to learn anatomy in undergraduate medicine? Med. Teach., 40(10):1020-9, 2018.

Ng, C. L.; Li, X.; Chee, S. C. \& Ngo, R. Y. An innovative 3-dimensional model of the epitympanum for teaching of middle ear anatomy. Otolaryngol. Head Neck Surg., 153(5):832-7, 2015.

Pujol, S.; Baldwin, M.; Nassiri, J.; Kinkinis, R. \& Shaffer, K. Using 3D modeling techniques to enhance teaching of difficult anatomical concepts. Acad. Radiol., 23(4):507-16, 2016.

Rovetto, P. Ideas Médicas: Una Mirada histórica. Cali, Universidad del Valle, 2008.

Dirección para correspondencia:

Sonia Osorio Toro

Departamento de Morfología

Grupo de Investigación TEBLAMI

Doctorando en Educación

Énfasis en Enseñanza de las Ciencias Naturales

Grupo de Investigación de Ciencias, acciones y creencias

Universidad del Valle

Calle 48 109-83

Cali

COLOMBIA

Email: sonia.osorio@correounivalle.edu.co

Recibido : $14-02-2020$

Aceptado: $30-03-2020$ 